VOL. 21, No. 2 (2017), 61-73

\title{
On the solutions of a second order difference equation
}

\author{
R. ABo-Zeid
}

Abstract. In this paper, we discuss the global behavior of all solutions of the difference equation

$$
x_{n+1}=\frac{x_{n} x_{n-1}}{a x_{n}+b x_{n-1}}, \quad n \in \mathbb{N}_{0},
$$

where $a, b$ are real numbers and the initial conditions $x_{-1}, x_{0}$ are real numbers.

We determine the forbidden set and give an explicit formula for the solutions. We show the existence of periodic solutions, under certain conditions.

\section{INTRODUCTION}

In this paper, we discuss the global behavior of the difference equation

$$
x_{n+1}=\frac{x_{n} x_{n-1}}{a x_{n}+b x_{n-1}}, \quad n \in \mathbb{N},
$$

where $a, b$ are real numbers and the initial conditions $x_{-1}, x_{0}$ are real numbers. Results concerning rational difference equations having quadratic terms are included in some publications such as [1]-[21] and the references cited therein.

Using the substitution $y_{n}=\frac{1}{x_{n}}$, we can obtain the linear second order homogeneous difference equation

$$
y_{n+1}=b y_{n}+a y_{n-1}, \quad n \in \mathbb{N}
$$

The characteristic equation of equation (2) is

$$
\lambda^{2}-b \lambda-a=0 \text {. }
$$

Equation (3) has two roots $\lambda_{-}=\frac{b}{2}-\frac{\sqrt{b^{2}+4 a}}{2}$ and $\lambda_{+}=\frac{b}{2}+\frac{\sqrt{b^{2}+4 a}}{2}$. The form of the solution should be according to the value of the quantity $b^{2}+4 a$.

The following theorem [12] is useful in studying the solutions of the difference equation (2).

2010 Mathematics Subject Classification. Primary: 39A20; Secondary: 39A21.

Key words and phrases. difference equation, forbidden set, periodic solution, unbounded solution. 
Theorem 1.1. The following statements holds:

(1) All solutions of (2) oscillates (about zero) if and only if the characteristic equation has no positive roots.

(2) All solutions of (2) converge to zero if and only if $\max \left\{\lambda_{1}, \lambda_{2}\right\}<1$.

In order to study the solutions of the difference equation (1), we consider the two cases:

- Case $a b>0$;

- Case $a b<0$.

The case $a b=0$ reduces equation (1) to the first order homogeneous difference equation $x_{n+1}=\frac{1}{b} x_{n}$ when $a=0$, and to the second order homogeneous difference equation $x_{n+1}=\frac{1}{a} x_{n-1}$ when $b=0$, which are easy to investigate.

\section{CASE $a b>0$}

In this section we discuss the behavior of the solutions when $a b>0$.

2.1. Case $a>0, b>0$. In this case, we have $b^{2}+4 a>0$ and so the roots $\lambda_{-}$and $\lambda_{+}$are real such that $\lambda_{-}<0<\frac{b}{2}<\lambda_{+}$. The solution of equation (2) is

$$
y_{n}=c_{1} \lambda_{-}^{n}+c_{2} \lambda_{+}^{n}, \quad n=-1,0,1, \ldots
$$

Using the initials $y_{-1}$ and $y_{0}$, the values of $c_{1}$ and $c_{2}$ are

$$
c_{1}=\frac{a\left(y_{0}-y_{-1} \lambda_{+}\right)}{\left(\lambda_{+}-\lambda_{-}\right) \lambda_{+}} \quad \text { and } \quad c_{2}=\frac{a\left(y_{-1} \lambda_{-}-y_{0}\right)}{\left(\lambda_{+}-\lambda_{-}\right) \lambda_{-}} .
$$

Then,

$$
y_{n}=\frac{1}{\lambda_{+}-\lambda_{-}}\left[y_{0}\left(\lambda_{+}^{n+1}-\lambda_{-}^{n+1}\right)+a y_{-1}\left(\lambda_{+}^{n}-\lambda_{-}^{n}\right)\right], \quad n=-1,0,1, \ldots
$$

If we put $y_{n}=0$, we get

$$
y_{0}=-a\left(\frac{\lambda_{+}^{n}-\lambda_{-}^{n}}{\lambda_{+}^{n+1}-\lambda_{-}^{n+1}}\right) y_{-1} .
$$

Hence, we conclude that, the forbidden set of equation (1) is

$$
F=\bigcup_{n=-1}^{\infty}\left\{\left(x_{0}, x_{-1}\right) \in \mathbb{R}^{2}: x_{0}=-\frac{1}{a}\left(\frac{\lambda_{+}^{n+1}-\lambda_{-}^{n+1}}{\lambda_{+}^{n}-\lambda_{-}^{n}}\right) x_{-1}\right\} .
$$

The solution of equation (1) is

$$
x_{n}=\frac{x_{0} x_{-1}\left(\lambda_{+}-\lambda_{-}\right)}{\left[x_{-1}\left(\lambda_{+}^{n+1}-\lambda_{-}^{n+1}\right)+a x_{0}\left(\lambda_{+}^{n}-\lambda_{-}^{n}\right)\right]}, \quad n=-1,0,1, \ldots
$$

Consider the set

$$
S=F \cup D,
$$


where $D=\left\{(u, v) \in \mathbb{R}^{2}: a u^{2}+b u v-v^{2}=0\right\}$. Note that for $(u, v) \in \mathbb{R}^{2}$, $c_{2}(u, v)=0$ implies that $a u^{2}+b u v-v^{2}=0$.

Theorem 2.1. Assume that $\left(x_{0}, x_{-1}\right) \notin S$ and let $\left\{x_{n}\right\}_{n=-1}^{\infty}$ be a solution of equation (1). Then the following statements are true.

(1) If $a+b<1$, then $\left\{x_{n}\right\}_{n=-1}^{\infty}$ is unbounded.

(2) If $a+b=1$, then $\left\{x_{n}\right\}_{n=-1}^{\infty}$ converges to $\frac{1}{c_{2}}$.

(3) If $a+b>1$, then $\left\{x_{n}\right\}_{n=-1}^{\infty}$ converges to zero.

Proof. Let $\left\{x_{n}\right\}_{n=-1}^{\infty}$ be a solution of equation (1) such that $\left(x_{0}, x_{-1}\right) \notin S$. When $a+b<1$, we have that $\lambda_{+}<1$. But $\left|\lambda_{-}\right|<\left|\lambda_{+}\right|$, then

$$
x_{n}=\frac{1}{c_{1} \lambda_{-}^{n}+c_{2} \lambda_{+}^{n}}=\frac{1}{\lambda_{+}^{n}\left(c_{1}\left(\frac{\lambda_{-}}{\lambda_{+}}\right)^{n}+c_{2}\right)} .
$$

That is

$$
x_{n} \rightarrow \infty\left(\operatorname{sgn}\left(c_{2}\right)\right) \text { as } n \rightarrow \infty,
$$

from which (1) follows.

When $a+b=1$, we have that $\lambda_{+}=1$. It follows that

$$
x_{n}=\frac{1}{c_{1} \lambda_{-}^{n}+c_{2}} \rightarrow \frac{1}{c_{2}} \quad \text { as } n \rightarrow \infty,
$$

from which (2) follows.

When $a+b>1$, we have that $\lambda_{+}>1$. Then

$$
x_{n}=\frac{1}{\lambda_{+}^{n}\left(c_{1}\left(\frac{\lambda_{-}}{\lambda_{+}}\right)^{n}+c_{2}\right)} \rightarrow 0 \text { as } n \rightarrow \infty,
$$

from which (3) follows.

Theorem 2.2. The subset

$$
D=\left\{(x, y) \in \mathbb{R}^{2}: a x^{2}+b x y-y^{2}=0\right\}
$$

is an invariant subset of the set $S$.

Proof. Let $\left(x_{0}, x_{-1}\right) \in D$. We show that $\left(x_{n}, x_{n-1}\right) \in D$ for each $n \in N$. The proof is by induction on $n$. For $n=0$, we have that

$$
a x_{0}^{2}+b x_{0} x_{-1}-x_{-1}^{2}=0 .
$$

This implies that

Now for $n=1$, we have

$$
a x_{0}+b x_{-1}=\frac{x_{-1}^{2}}{x_{0}} .
$$

$$
\begin{gathered}
a x_{1}^{2}+b x_{1} x_{0}-x_{0}^{2}=a \frac{x_{0}^{2} x_{-1}^{2}}{\left(a x_{0}+b x_{-1}\right)^{2}}+b x_{0} \frac{x_{0} x_{-1}}{a x_{0}+b x_{-1}}-x_{0}^{2} \\
=a \frac{x_{0}^{4}}{x_{-1}^{2}}+b \frac{x_{0}^{3}}{x_{-1}}-x_{0}^{2}=\frac{x_{0}^{2}}{x_{-1}^{2}}\left(a x_{0}^{2}+b x_{0} x_{-1}-x_{-1}^{2}\right)=0 .
\end{gathered}
$$


This implies that $\left(x_{1}, x_{0}\right) \in D$.

Suppose that the relation is true for $n=k$. That is $\left(x_{k}, x_{k-1}\right) \in D$. Then

$$
a x_{k}+b x_{k-1}=\frac{x_{k-1}^{2}}{x_{k}} .
$$

That is

$$
\begin{gathered}
a x_{k+1}^{2}+b x_{k+1} x_{k}-x_{k}^{2}=a \frac{x_{k}^{2} x_{k-1}^{2}}{\left(a x_{k}+b x_{k-1}\right)^{2}}+b x_{k} \frac{x_{k} x_{k-1}}{a x_{k}+b x_{k-1}}-x_{k}^{2} \\
=a \frac{x_{k}^{4}}{x_{k-1}^{2}}+b \frac{x_{k}^{3}}{x_{k-1}}-x_{k}^{2}=\frac{x_{k}^{2}}{x_{k-1}^{2}}\left(a x_{k}^{2}+b x_{k} x_{k-1}-x_{k-1}^{2}\right)=0 .
\end{gathered}
$$

Therefore, $\left(x_{k+1}, x_{k}\right) \in D$.

This completes the proof.

Theorem 2.3. Assume that $b=a-1$ and let $\left(x_{0}, x_{-1}\right) \notin F$. If $c_{2}=0$, then $\left\{x_{n}\right\}_{n=-1}^{\infty}$ is periodic with prime period two.

Proof. Clear that if $b=a-1$, then $\lambda_{-}=-1$. It follows that

$$
x_{n}=\frac{1}{c_{1}(-1)^{n}+c_{2} \lambda_{+}^{n}} .
$$

But as $c_{2}=0$, we have that $x_{0}=-x_{-1}$ and so $c_{1}=\frac{1}{x_{0}}$. This implies that

$$
x_{n}=\frac{1}{c_{1}(-1)^{n}}=\left\{\begin{array}{rr}
\frac{1}{c_{1}}=x_{0}, & n \text { even, } \\
-\frac{1}{c_{1}}=-x_{0}, & n \text { odd } .
\end{array}\right.
$$

This completes the proof.

2.2. Case $a<0, b<0$. In this subsection, suppose that both $a$ and $b$ are negative. If $b^{2}+4 a>0$, the two roots $\lambda_{-}$and $\lambda_{+}$are also negative such that $\lambda_{-}<\frac{b}{2}<\lambda_{+}<0$.

Theorem 2.4. Assume that $\left(x_{0}, x_{-1}\right) \notin S$ and let $\left\{x_{n}\right\}_{n=-1}^{\infty}$ be a solution of equation (1). Then the following statements are true.

(1) If $b<a-1$, then $\left\{x_{n}\right\}_{n=-1}^{\infty}$ is converges to zero.

(2) If $b=a-1$, then we have the following:

(a) If $a \leq-1$, then $\left\{x_{n}\right\}_{n=-1}^{\infty}$ converges to zero.

(b) If $a>-1$, then $\left\{x_{n}\right\}_{n=-1}^{\infty}$ converges to a period-2 solution.

(3) If $-2 \sqrt{-a}>b>a-1$, then we have the following:

(a) If $a>-1$, then $\left\{x_{n}\right\}_{n=-1}^{\infty}$ is unbounded.

(b) If $a<-1$, then $\left\{x_{n}\right\}_{n=-1}^{\infty}$ converges to zero.

Proof. Let $\left\{x_{n}\right\}_{n=-1}^{\infty}$ be a solution of equation (1) such that $\left(x_{0}, x_{-1}\right) \notin S$. When $b<a-1$, we have that $\lambda_{-}<-1<\lambda_{+}<0$. Then

$$
x_{n}=\frac{1}{\lambda_{-}^{n}\left(c_{1}+c_{2}\left(\frac{\lambda_{+}}{\lambda_{-}}\right)^{n}\right)} \rightarrow 0 \text { as } n \rightarrow \infty,
$$


from which (1) follows.

When $b=a-1$, we have that $\lambda=-1$ is a root of equation (3).

If $a=-1$, then $\lambda=-1$ is a repeated root and then $\left\{x_{n}\right\}_{n=-1}^{\infty}$ converges to zero.

Now suppose that $a \neq-1$. If $a<-1$, then $\lambda_{-}=-1$ and $\lambda_{+}=a$, from which (2a) follows. Similarly, when $a>-1, \lambda_{-}=a$ and $\lambda_{+}=-1$, from which $(2 \mathrm{~b})$ follows.

When $b>a-1$, we have either $\lambda_{-}<\lambda_{+}<-1$ or $-1<\lambda_{-}<\lambda_{+}<0$. If $a>-1$, then $-1<\lambda_{-}<\lambda_{+}<0$ from which (3a) follows. If $a<-1$, then $\lambda_{-}<\lambda_{+}<-1$ from which (3b) follows. This completes the proof.

Theorem 2.5. Assume that $a<0$ and $b<0$ and let $\left(x_{0}, x_{-1}\right) \notin F$. The following statements hold:

(1) If $b=a-1$, then there exist periodic solutions of prime period two.

(2) All solutions of equation (1) oscillate about zero

Proof. (1) Suppose that $b=a-1$ and let $\left(x_{0}, x_{-1}\right) \notin F$. If $a>-1$ let Then

$$
x_{n}=\frac{1}{c_{1}(-1)^{n}+c_{2} \lambda_{+}^{n}} .
$$

If $c_{2}=0$, we have that $x_{0}=-x_{-1}$. Then $c_{1}=\frac{1}{x_{0}}$. This implies that

$$
x_{n}=\frac{1}{c_{1}(-1)^{n}}=\left\{\begin{array}{rr}
\frac{1}{c_{1}}=x_{0}, & n \text { even, } \\
-\frac{1}{c_{1}}=-x_{0}, & n \text { odd } .
\end{array}\right.
$$

If $a<-1$, then

$$
x_{n}=\frac{1}{c_{1} \lambda_{-}^{n}+c_{2}(-1)^{n}} .
$$

If $c_{1}=0$, then $c_{2}=\frac{1}{x_{0}}$ and therefore, $\left\{x_{n}\right\}_{n=-1}^{\infty}$ is periodic with prime period two.

(2) Clear that $a<0$ and $b<0$ implies negative roots for equation (3). Using Theorem (1.1), we get the result.

The rest of this subsection is devoted to discuss the case $b^{2}+4 a \leq 0$. When $b^{2}+4 a=0$ the solution of equation (2) is

$$
y_{n}=c_{1}\left(\frac{b}{2}\right)^{n}+c_{2}\left(\frac{b}{2}\right)^{n} n, \quad n=-1,0,1, \ldots
$$

By a simple calculations, we can obtain the solution

$$
x_{n}=\frac{x_{0} x_{-1}}{\left(\frac{b}{2}\right)^{n}\left(-\frac{b}{2} x_{0} n+(1+n) x_{-1}\right)}, \quad n=-1,0,1, \ldots
$$

The forbidden set $F$ of equation (1) in this case is

$$
F=\bigcup_{n=-1}^{\infty}\left\{\left(x_{0}, x_{-1}\right) \in \mathbb{R}^{2}: x_{0}=\frac{2(1+n) x_{-1}}{b n}\right\} \text {. }
$$


Similarly, when $b^{2}+4 a<0$, the solution of equation (2) is

$$
x_{n}=\frac{x_{0} x_{-1} \sin \theta}{(-a)^{\frac{n}{2}}\left(x_{-1} \sin (n+1) \theta-\sqrt{-a}\left(x_{0} \sin n \theta\right)\right)},
$$

where $\left.\theta=\tan ^{-1} \frac{\sqrt{-b^{2}-4 a}}{b} \in\right] \frac{\pi}{2}, \pi[$.

The forbidden set $F$ of equation (1) in this case is

$$
F=\bigcup_{n=-1}^{\infty}\left\{\left(x_{0}, x_{-1}\right) \in \mathbb{R}^{2}: x_{0}=\frac{x_{-1} \sqrt{-b^{2}-4 a}}{-2 a}\left(\frac{b}{\sqrt{-b^{2}-4 a}}+\cot n \theta\right)\right\} \text {. }
$$

Theorem 2.6. Assume that $b=-2 \sqrt{-a}$ and let $\left\{x_{n}\right\}_{n=-1}^{\infty}$ be a solution of equation (1) such that $\left(x_{0}, x_{-1}\right) \notin S$. The following statements are true.

(1) If $a \leq-1$, then $\left\{x_{n}\right\}_{n=-1}^{\infty}$ converges to zero.

(2) If $a>-1$, then $\left\{x_{n}\right\}_{n=-1}^{\infty}$ is unbounded.

Proof. Let $\left\{x_{n}\right\}_{n=-1}^{\infty}$ be a solution of equation (1) such that $\left(x_{0}, x_{-1}\right) \notin S$. If $b=-2 \sqrt{-a}$, then $\lambda=-\sqrt{-a}$ is a repeated root of equation (3). That is

$$
x_{n}=\frac{1}{c_{1}(-a)^{\frac{n}{2}}+c_{2}(-a)^{\frac{n}{2}} n} .
$$

When $a \leq-1$, we have that $(-a)^{n} n$ diverges to $\infty$ as $n \rightarrow \infty$. Then $\left\{x_{n}\right\}_{n=-1}^{\infty}$ converges to zero, from which (1) follows. When $a>-1$, we have that $(-a)^{n} n$ converges to zero, from which (2) follows.

Theorem 2.7. Assume that $0>b>-2 \sqrt{-a}$ and let $\left\{x_{n}\right\}_{n=-1}^{\infty}$ be a solution of equation (1) such that $\left(x_{0}, x_{-1}\right) \notin S$. The following statements are true.

(1) If $a<-1$, then $\left\{x_{n}\right\}_{n=-1}^{\infty}$ converges to zero.

(2) If $a=-1$, then $\left\{x_{n}\right\}_{n=-1}^{\infty}$ is bounded.

(3) If $a>-1$, then $\left\{x_{n}\right\}_{n=-1}^{\infty}$ is unbounded.

Proof. Let $\left\{x_{n}\right\}_{n=-1}^{\infty}$ be a solution of equation (1) such that $\left(x_{0}, x_{-1}\right) \notin S$. If $0>b>-2 \sqrt{-a}$, then the roots of equation (3) are complex and $\left|\lambda_{ \pm}\right|=$ $\sqrt{-a}$. Then

$$
x_{n}=\frac{1}{(-a)^{\frac{n}{2}}\left(c_{1} \cos n \theta+c_{2} \sin n \theta\right)},
$$

where $\left.\theta=\tan ^{-1} \frac{\sqrt{-b^{2}-4 a}}{b} \in\right] \frac{\pi}{2}, \pi\left[\right.$. When $a<-1$, we have that $(-a)^{n}$ diverges to $\infty$ as $n \rightarrow \infty$. Then $\left\{x_{n}\right\}_{n=-1}^{\infty}$ converges to zero, from which (1) follows. When $a=-1$, we have that

$$
x_{n}=\frac{1}{c_{1} \cos n \theta+c_{2} \sin n \theta},
$$

$\theta=\tan ^{-1} \frac{\sqrt{-b^{2}+4}}{b}$. As $\left(x_{0}, x_{-1}\right) \notin F$, we have that for any $n \in \mathbb{N}$

$$
c_{1} \cos n \theta+c_{2} \sin n \theta \neq 0 \text {. }
$$


This means that, there exists $\epsilon>0$ such that $\left|c_{1} \cos n \theta+c_{2} \sin n \theta\right|>\epsilon$, from which (2) follows. When $a>-1$, we have that $(-a)^{n}$ converges to zero, from which (3) follows.

Theorem 2.8. Assume that $a=-1$ and $0>b>-2$, and let $\left\{x_{n}\right\}_{n=-1}^{\infty}$ be a solution of equation (1) such that $\left(x_{0}, x_{-1}\right) \notin F$. If $\theta=\frac{p}{q} \pi$, where $p$ and $q$ are positive relatively prime integers such that $\frac{q}{2}<p<q$, then $\left\{x_{n}\right\}_{n=-1}^{\infty}$ is a periodic solution with prime period $2 q$.

Proof. Let $\left\{x_{n}\right\}_{n=-1}^{\infty}$ be a solution of equation (1) such that $\left(x_{0}, x_{-1}\right) \notin F$. Clear that, if $a=-1$ and $-2<b<0$, the angle $\left.\theta=\frac{p}{q} \pi \in\right] \frac{\pi}{2}, \pi[$.

The solution (7) becomes

$$
x_{n}=\frac{x_{0} x_{-1} \sin \theta}{\left(x_{-1} \sin (n+1) \theta-x_{0} \sin n \theta\right)} .
$$

Then

$$
\begin{aligned}
x_{n+2 q} & =\frac{x_{0} x_{-1} \sin \theta}{\left(x_{-1} \sin (n+2 q+1) \theta-x_{0} \sin (n+2 q) \theta\right)} \\
& =\frac{x_{0} x_{-1} \sin \theta}{\left(x_{-1} \sin ((n+1) \theta+2 q \theta)-x_{0} \sin (n \theta+2 q \theta)\right.} \\
& =\frac{x_{0} x_{-1} \sin \theta}{\left(x_{-1} \sin ((n+1) \theta+2 p \pi)-x_{0} \sin (n \theta+2 p \pi)\right.} \\
& =x_{n} .
\end{aligned}
$$

This completes the proof.

Example (1). Figure 1. shows that if $a=0.2, b=0.8(a+b=1)$. Then the solution $\left\{x_{n}\right\}_{n=-1}^{\infty}$ of equation (1) with initial conditions $x_{-1}=-0.5$ and $x_{0}=1$ converges to $\frac{1}{c_{2}}=2$.

Example (2). Figure 2. shows that if $a=2, b=1,(b=a-1)$. Then the solution $\left\{x_{n}\right\}_{n=-1}^{\infty}$ of equation (1) with initial conditions $x_{-1}=-2$ and $x_{0}=2\left(c_{2}=0\right)$ is periodic with prime period two.

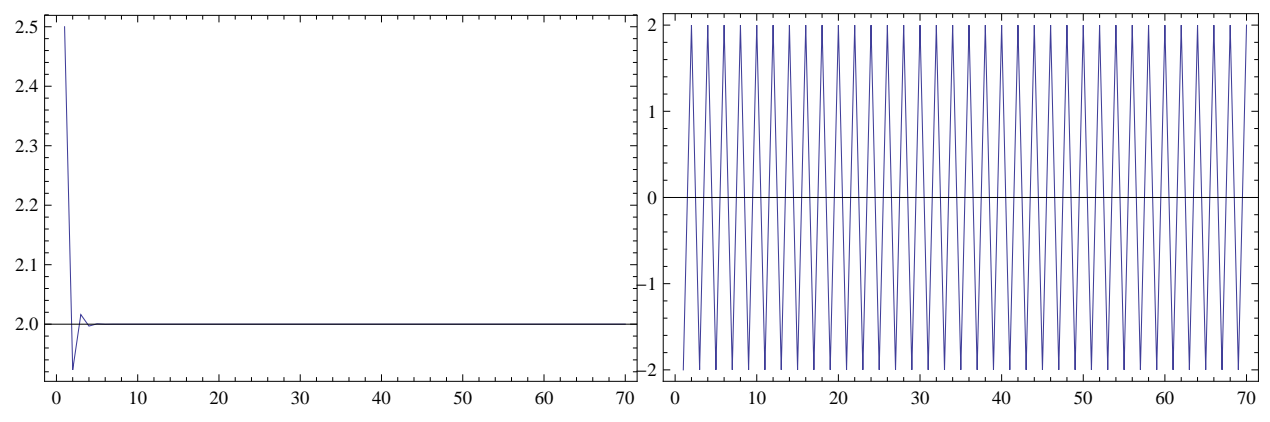

FIGURE 1. $x_{n+1}=\frac{x_{n} x_{n-1}}{0.2 x_{n}+0.8 x_{n-1}}$.

FIGURE 2. $x_{n+1}=\frac{x_{n} x_{n-1}}{2 x_{n}+x_{n-1}}$. 


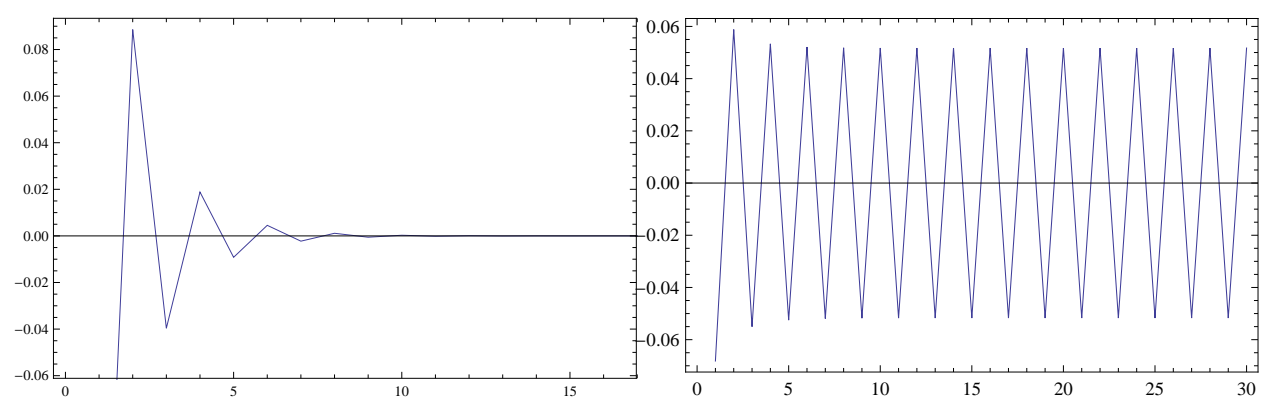

FiguRE 3. $x_{n+1}=\frac{x_{n} x_{n-1}}{-2 x_{n}-3 x_{n-1}}$. FigURE 4. $x_{n+1}=\frac{x_{n} x_{n-1}}{-0.5 x_{n}-1.5 x_{n-1}}$.

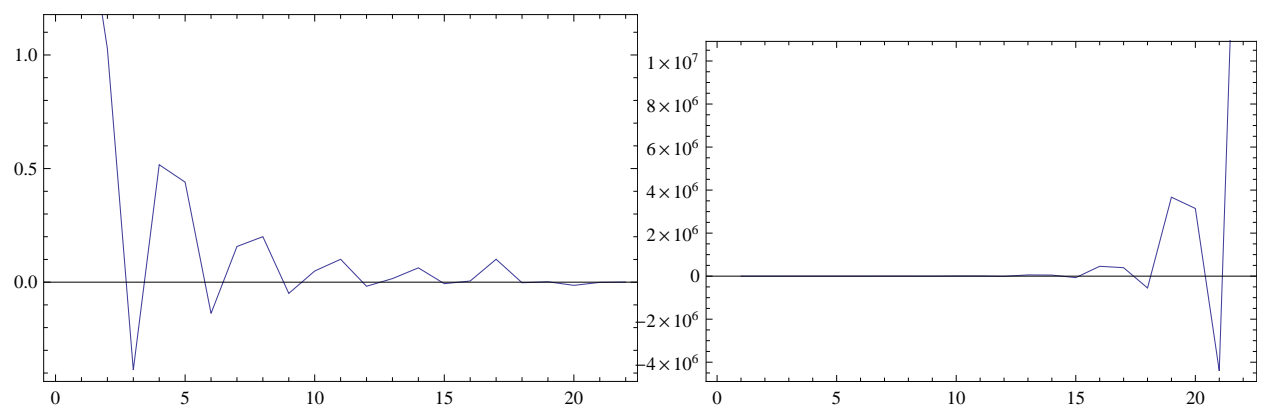

FiguRE 5. $x_{n+1}=\frac{x_{n} x_{n-1}}{-2 x_{n}-1.5 x_{n-1}}$. FiguRE 6. $x_{n+1}=\frac{x_{n} x_{n-1}}{-0.25 x_{n}-0.5 x_{n-1}}$.

Example (3). Figure 3. shows that if $a=-2, b=-3(b=a-1)$. Then the solution $\left\{x_{n}\right\}_{n=-1}^{\infty}$ of equation (1) with initial conditions $x_{-1}=1.1$ and $x_{0}=1.2$ converges to zero.

Example (4). Figure 4. shows that if $a=-0.5, b=-1.5,(b=a-1)$. Then the solution $\left\{x_{n}\right\}_{n=-1}^{\infty}$ of equation (1) with initial conditions $x_{-1}=-1.5$ and $x_{0}=0.1$ converges to a period- 2 solution.

Example (5). Figure 5. shows that if $a=-2, b=-1.5(0>b>-2 \sqrt{-a} \approx$ -2.8284 and $a<-1)$. Then the solution $\left\{x_{n}\right\}_{n=-1}^{\infty}$ of equation (1) with initial conditions $x_{-1}=2.5$ and $x_{0}=-1.1$ converges to zero.

Example (6). Figure 6. shows that if $a=-0.25, b=-0.5,0>b>$ $-2 \sqrt{-a}=-1$ and $a>-1$ ). Then the solution $\left\{x_{n}\right\}_{n=-1}^{\infty}$ of equation (1) with initial conditions $x_{-1}=1.5$ and $x_{0}=-2.1$ is unbounded.

Example (7). Figure 7. shows that if $a=-1, b=-\frac{5-\sqrt{5}}{2}\left(b^{2}+4 a<0\right)$. Then the solution $\left\{x_{n}\right\}_{n=-1}^{\infty}$ of equation (1) with initial conditions $x_{-1}=2.7$ and $x_{0}=-0.4$ is periodic with prime period 20. Note that $\theta=\frac{7}{10} \pi$. 
Example (8). Figure 8. shows that if $a=-1, b=-\frac{3-\sqrt{5}}{2}\left(b^{2}+4 a<0\right)$. Then the solution $\left\{x_{n}\right\}_{n=-1}^{\infty}$ of equation (1) with initial conditions $x_{-1}=$ -2.2 and $x_{0}=-0.5$ is periodic with prime period 10 . Note that $\theta=\frac{3}{5} \pi$.

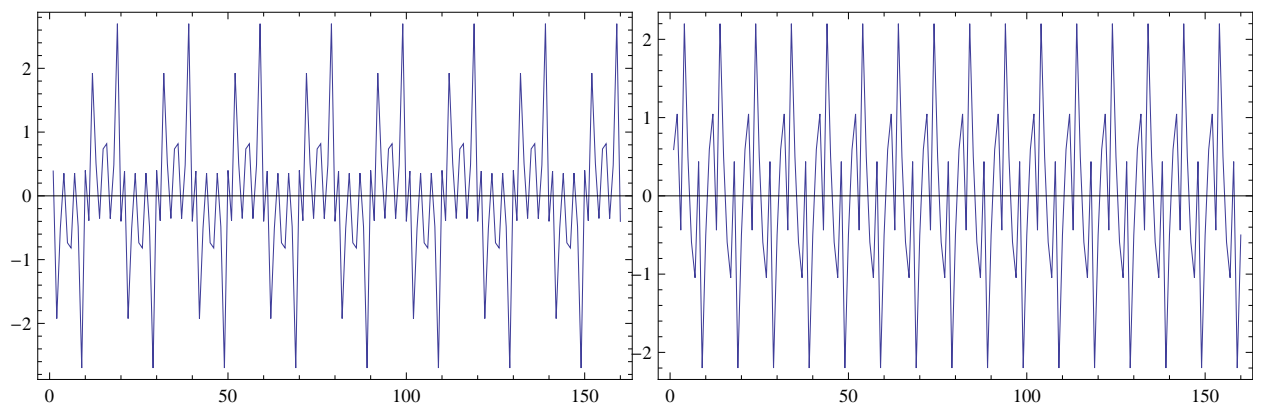

FiguRE 7. $x_{n+1}=\frac{x_{n} x_{n-1}}{-x_{n}-\frac{5-\sqrt{5}}{2} x_{n-1}}$. FigURE 8. $x_{n+1}=\frac{x_{n} x_{n-1}}{-x_{n}-\frac{3-\sqrt{5}}{2} x_{n-1}}$.

\section{CASE $a b<0$}

3.1. Case $a>0$ and $b<0$. In this case $b^{2}+4 a>0$, that is the two roots $\lambda_{-}$and $\lambda_{+}$are real such that $\lambda_{-}<\frac{b}{2}<0<\lambda_{+}$and $\left|\lambda_{-}\right|>\left|\lambda_{+}\right|$.

Theorem 3.1. Assume that $\left(x_{0}, x_{-1}\right) \notin S$ and let $\left\{x_{n}\right\}_{n=-1}^{\infty}$ be a solution of equation (1). The following statements are true.

(1) If $b<a-1$, then $\left\{x_{n}\right\}_{n=-1}^{\infty}$ is converges to zero.

(2) If $b=a-1$, then $\left\{x_{n}\right\}_{n=-1}^{\infty}$ converges to a period-2 solution.

(3) If $b>a-1$, then $\left\{x_{n}\right\}_{n=-1}^{\infty}$ is unbounded.

Proof. Let $\left\{x_{n}\right\}_{n=-1}^{\infty}$ be a solution of equation (1) such that $\left(x_{0}, x_{-1}\right) \notin S$. When $b<a-1$, we have that $\lambda_{-}<-1$. It follows that

$$
x_{n}=\frac{1}{\lambda_{-}^{n}\left(c_{1}+c_{2}\left(\frac{\lambda_{+}}{\lambda_{-}}\right)^{n}\right)} \rightarrow 0 \text { as } n \rightarrow \infty,
$$

from which (1) follows.

When $b=a-1$, we have that $\lambda_{-}=-1$. Then $x_{n}$ converges to the period-2 solution $\left\{\ldots, \frac{1}{c_{1}},-\frac{1}{c_{1}}, \frac{1}{c_{1}},-\frac{1}{c_{1}}, \ldots\right\}$, from which (2) follows.

When $b>a-1$, we have that $0>\lambda_{-}>-1$. If $a>-1$, then $-1<\lambda_{-}<$ $\lambda_{+}<0$ from which (3) follows.

This completes the proof.

Theorem 3.2. Assume that $b=a-1$. Then there exist periodic solutions of prime period two.

Proof. Assume that $b=a-1$ and let $\left(x_{0}, x_{-1}\right) \notin F$. It is sufficient to see that if $c_{2}=0$, then $c_{1}=\frac{1}{x_{0}}$ and the result follows. 
3.2. Case $a<0$ and $b>0$. In this case, $b^{2}+4 a$ can be negative, zero or a positive real number. If $b^{2}+4 a>0$, then the roots of equation (3) are positive such that $0<\lambda_{-}<\lambda_{+}$.

Theorem 3.3. Assume that $\left(x_{0}, x_{-1}\right) \notin S$ and let $\left\{x_{n}\right\}_{n=-1}^{\infty}$ be a solution of equation (1). The following statements are true.

(1) If $b>1-a$, then $\left\{x_{n}\right\}_{n=-1}^{\infty}$ is converges to zero.

(2) If $b=1-a$, then we have the following:

(a) If $a>-1$, then $\left\{x_{n}\right\}_{n=-1}^{\infty}$ converges to $\frac{1}{c_{2}}$.

(b) If $a<-1$, then $\left\{x_{n}\right\}_{n=-1}^{\infty}$ converges to zero.

(3) If $2 \sqrt{-a}<b<1-a$, then we have the following:

(a) If $a>-1$, then $\left\{x_{n}\right\}_{n=-1}^{\infty}$ is unbounded.

(b) If $a<-1$, then $\left\{x_{n}\right\}_{n=-1}^{\infty}$ converges to zero.

Proof. Let $\left\{x_{n}\right\}_{n=-1}^{\infty}$ be a solution of equation (1) such that $\left(x_{0}, x_{-1}\right) \notin S$. When $b>1-a$, we have that $\lambda_{-}<1<\lambda_{+}$. It follows that

$$
x_{n}=\frac{1}{\lambda_{+}^{n}\left(c_{1}\left(\frac{\lambda_{-}}{\lambda_{+}}\right)^{n}+c_{2}\right)} \rightarrow 0 \text { as } n \rightarrow \infty,
$$

from which (1) follows.

When $a+b=1, \lambda=1$ is a root for equation (3).

If $a>-1$, then $\lambda_{-}=-a$ and $\lambda_{+}=1$. Now

$$
x_{n}=\frac{1}{\left(c_{1} \lambda_{-}^{n}+c_{2}\right)} \rightarrow \frac{1}{c_{2}} \text { as } n \rightarrow \infty,
$$

from which (2a) follows. If $a<-1$, then $\lambda_{-}=1$ and $\lambda_{+}=-a$, from which (2b) follows.

Note that, when $a+b=1, b^{2}+4 a>0$ when $a \neq-1$.

When $2 \sqrt{-a}<b<1-a$, we have either $0<\lambda_{-}<\lambda_{+}<1$ or $1<\lambda_{-}<\lambda_{+}$. If $a>-1$, we have that $0<\lambda_{-}<\lambda_{+}<1$, from which (3a) follows.

If $a<-1$, then $1<\lambda_{-}<\lambda_{+}$, from which (3b) follows.

This completes the proof.

Theorem 3.4. Assume that $0<b<2 \sqrt{-a}$ and let $\left\{x_{n}\right\}_{n=-1}^{\infty}$ be a solution of equation (1) such that $\left(x_{0}, x_{-1}\right) \notin S$. The following statements are true.

(1) If $a<-1$, then $\left\{x_{n}\right\}_{n=-1}^{\infty}$ converges to zero.

(2) If $a=-1$, then $\left\{x_{n}\right\}_{n=-1}^{\infty}$ is bounded.

(3) If $a>-1$, then $\left\{x_{n}\right\}_{n=-1}^{\infty}$ is unbounded.

Proof. Let $\left\{x_{n}\right\}_{n=-1}^{\infty}$ be a solution of equation (1) such that $\left(x_{0}, x_{-1}\right) \notin S$. It is sufficient to see that, if $0<b<2 \sqrt{-a}$, then the roots of equation (3) are complex such that $\left|\lambda_{ \pm}\right|=\sqrt{-a}$ and $\left.\theta=\tan ^{-1} \frac{\sqrt{-b^{2}-4 a}}{b} \in\right] 0, \frac{\pi}{2}$ [. The rest of the proof is similar to that of Theorem (2.7) and will be omitted.

Theorem 3.5. Assume that $a=-1$ and $0>b>-2$, and let $\left\{x_{n}\right\}_{n=-1}^{\infty}$ be a solution of equation (1) such that $\left(x_{0}, x_{-1}\right) \notin F$. If $\theta=\frac{p}{q} \pi$, where $p$ and 
$q$ are positive relatively prime integers such that $0<p<\frac{q}{2}$, then $\left\{x_{n}\right\}_{n=-1}^{\infty}$ is a periodic solution with prime period $2 q$.

Proof. The proof is similar to that of Theorem (2.8) and will be omitted.

Example (9). Figure 9. shows that if $a=0.9, b=-0.1(b=a-1)$. Then the solution $\left\{x_{n}\right\}_{n=-1}^{\infty}$ of equation (1) with initial conditions $x_{-1}=2.5$ and $x_{0}=2.1$ converges to period- 2 solution.

Example (10). Figure 10. shows that if $a=-0.25, b=1.1,(1=2 \sqrt{-a}<$ $b<1-a=1.25)$. Then the solution $\left\{x_{n}\right\}_{n=-1}^{\infty}$ of equation (1) with initial conditions $x_{-1}=2.5$ and $x_{0}=-1.1$ is unbounded.

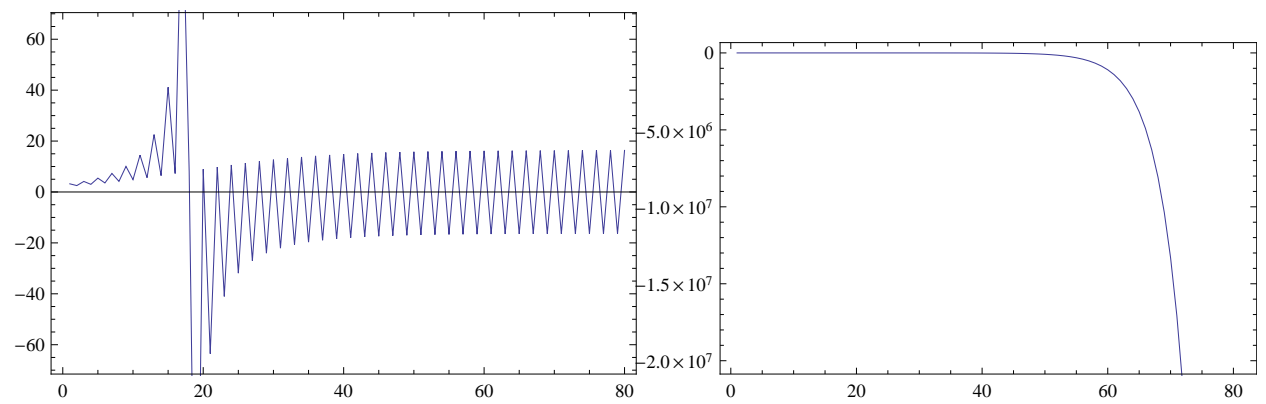

Figure 9. $x_{n+1}=\frac{x_{n} x_{n-1}}{0.9 x_{n}-0.1 x_{n-1}}$. Figure 10. $x_{n+1}=\frac{x_{n} x_{n-1}}{-0.25 x_{n}+1.1 x_{n-1}}$.

Example (11). Figure 11. shows that if $a=-1, b=-1\left(b^{2}+4 a<0\right)$. Then the solution $\left\{x_{n}\right\}_{n=-1}^{\infty}$ of equation (1) with initial conditions $x_{-1}=2.5$ and $x_{0}=-1.1$ is a period- 3 solution.

Example (12). Figure 12. shows that if $a=-1, b=1,\left(b^{2}+4 a<0\right)$. Then the solution $\left\{x_{n}\right\}_{n=-1}^{\infty}$ of equation (1) with initial conditions $x_{-1}=2.5$ and $x_{0}=-1.1$ is a period- 6 solution.

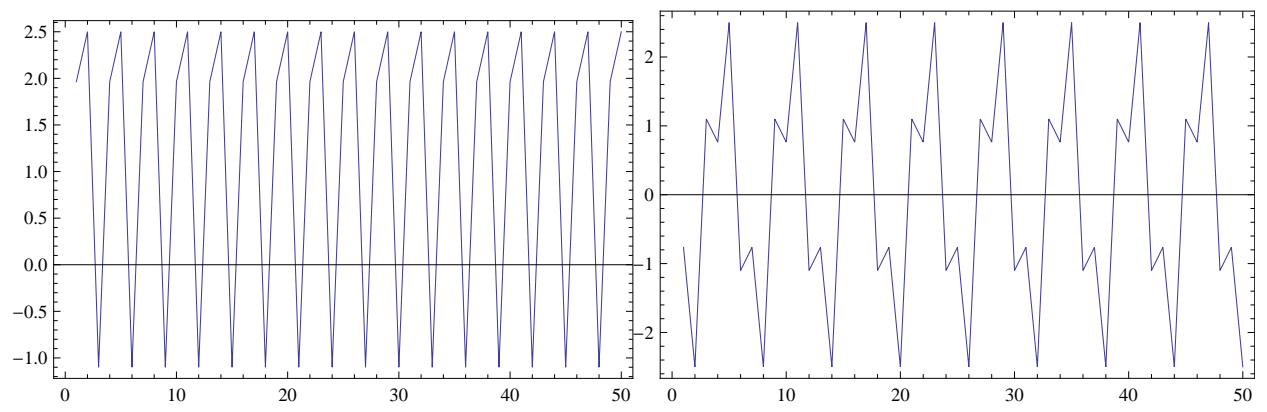

FIGURE 11. $x_{n+1}=\frac{x_{n} x_{n-1}}{-x_{n}-x_{n-1}}$.

FIGURE 12. $x_{n+1}=\frac{x_{n} x_{n-1}}{-x_{n}+x_{n-1}}$. 


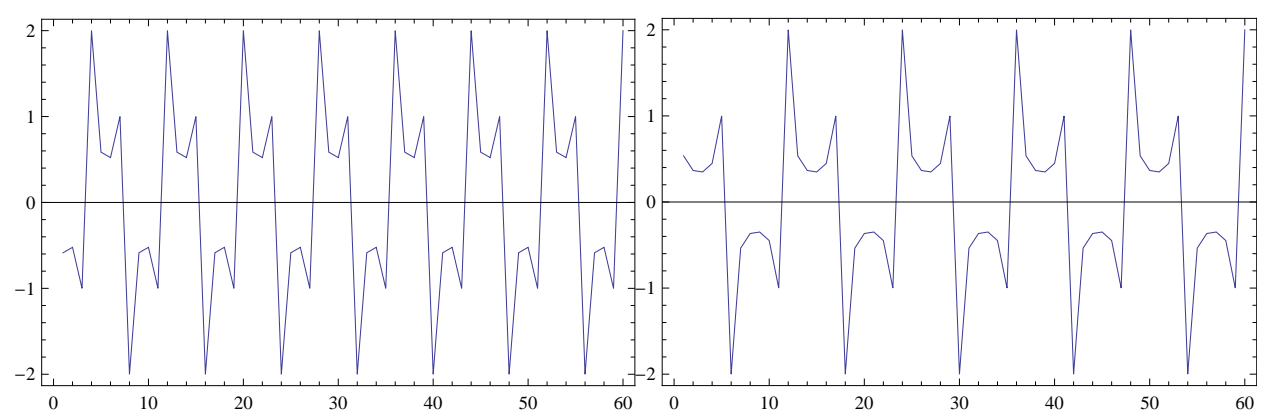

FiguRE 13. $x_{n+1}=\frac{x_{n} x_{n-1}}{-x_{n}+\sqrt{2} x_{n-1}}$. FigURE 14. $x_{n+1}=\frac{x_{n} x_{n-1}}{-x_{n}+\sqrt{3} x_{n-1}}$.

Example (13). Figure 13. shows that if $a=-1, b=\sqrt{2}\left(b^{2}+4 a<0\right)$. Then the solution $\left\{x_{n}\right\}_{n=-1}^{\infty}$ of equation (1) with initial conditions $x_{-1}=1$ and $x_{0}=-2$ is periodic with prime period 8 . Note that $\theta=\frac{1}{4} \pi$.

Example (14). Figure 14. shows that if $a=-1, b=\sqrt{3}\left(b^{2}+4 a<0\right)$. Then the solution $\left\{x_{n}\right\}_{n=-1}^{\infty}$ of equation (1) with initial conditions $x_{-1}=-1$ and $x_{0}=2$ is periodic with prime period 12 . Note that $\theta=\frac{1}{6} \pi$.

\section{REFERENCES}

[1] R. Abo-Zeid, Global behavior of a third order rational difference equation, Math. Bohem., 139 (1) (2014), 25 - 37.

[2] R. Abo-Zeid, Global behavior of a rational difference equation with quadratic term, Math. Morav., 18 (1) (2014), $81-88$.

[3] R. Abo-Zeid, On the solutions of two third order recursive sequences, Armenian J. Math., 6 (2) (2014), $64-66$.

[4] R. Abo-Zeid, Global behavior of a fourth order difference equation, Acta Comment. Univ. Tartu. Math., 18 (2) (2014), $211-220$.

[5] A.M. Amleh, E. Camouzis, and G. Ladas, On the dynamics of a rational difference equations, part 1. Int. J. Difference Equ., 3(1) (2008), 1 - 35.

[6] A.M. Amleh, E. Camouzis, and G. Ladas, On the dynamics of a rational difference equations, part 2. Int. J. Difference Equ., 3(2) (2008), 195 - 225.

[7] M.A. Al-Shabi, R. Abo-Zeid, Global Asymptotic Stability of a Higher Order Difference Equation, Appl. Math. Sci., 4 (17) (2010), 839 - 847.

[8] I. Bajo and E. Liz, Global behaviour of second-order nonlinear difference equation, J. Difference Equ. Appl., 17(10) (2011), 1471 - 1486.

[9] K. S. Berenhaut, J. D. Foley, S. Stevic, the global attractivity of the rational difference equation $y_{n+1}=\frac{y_{n-k}+y_{n-m}}{1+y_{n-k} y_{n-m}}$, Appl. Math. Lett., 20 (1) (2007), $54-58$. 
[10] E. Camouzis, G. Ladas, I. W. Rodrigues and S. Northshield, On the rational recursive sequence $x_{n+1}=\frac{\gamma x_{n}^{2}}{1+x_{n-1}^{2}}$, Comput. Math. Appl. 28 (1-3) (1994), $37-43$.

[11] M. Dehghan, C. M. Kent, R. Mazrooei-Sebdani, N. L. Ortiz, H. Sedaghat, Dynamics of rational difference equations containing quadratic terms, J. Difference Equ. Appl. 14 (2) (2008), $191-208$.

[12] S. Elaydi, An Introduction to Difference Equations, Third Edition, Springer, New York, 2005.

[13] E. A. Grove, E. J. Janowski, C. M. Kent, G. Ladas, On the rational recursive sequence $x_{n+1}=\frac{\alpha x_{n}+\gamma}{\left(\gamma x_{n}+\delta\right) x_{n-1}}$, Comm. Appl. Nonlinear Anal., 1 (3) (1994), $61-72$.

[14] M.R.S. Kulenovic and M. Mehuljic. Global behavior of some rational second order difference equations, Int. J. Difference Equ., 7(2) (2012), 153 - 162.

[15] R. Mazrooei-Sebdanti. Chaos in rational systems in the plane containing quadratic terms, Commun. Nonlinear Sci. Numer. Simulat., 17 (2012), 3857 - 3865.

[16] H. Sedaghat, Global behaviours of rational difference equations of orders two and three with quadratic terms, J. Diff. Eq. Appl., 15 (3) (2009), 215 - 224.

[17] S. Stevic, Global stability and asymptotics of some classes of rational difference equations, J. Math. Anal. Appl. 316 (1) (2006), 60 - 68.

[18] L. Xianyi and Z. Deming. Global asymptotic stability in a rational equation, J. Difference Equ. Appl., 9(9) (2003), 833 - 839

[19] X. Yang, D. J. Evans and G. M. Megson, On two rational difference equations, Appl. Math. Comput. 176 (2) (2006), $422-430$.

[20] X. Yang, On the global asymptotic stability of the difference equation $x_{n+1}=$ $\frac{x_{n-1} x_{n-1} x_{n-2}+x_{n-3}+\alpha}{x_{n-1}+x_{n-2} x_{n-3}+\alpha}$, Appl. Math. Comput. 171 (2) (2005), $857-861$.

[21] X.Yang, W.Su, B.Chen, G.M.Megson, D.J.Evans, On the recursive sequence $x_{n+1}=$ $\frac{a x_{n}+b x_{n-1}}{c+d x_{n} x_{n-1}}$, Appl. Math. Comput., 162 (2005) $1485-1497$.

\author{
R. Abo-Zeid \\ Department of Basic Science \\ The Higher Institute For \\ Engineering \& Technology- Al-Obour \\ CAIRO \\ EGYPT \\ E-mail address: abuzead73@yahoo.com
}

